\title{
La (de)seguritización del Gobierno Regional del Kurdistán. El movimiento kurdo en Iraq desde $1991^{1}$
}

The (de)securtization of the Kurdistan Regional Government. The Kurdish movement in Iraq since 1991.

\section{Juan Carlos CASTILLO QUIÑONES}

Universidad Nacional Autónoma de México

juancastillo@politicas.unam.mx

https://orcid.org/0000-0003-3942-2342

Recibido 22/08/2019. Revisado y aceptado para publicación 13/12/2019

Para citar este artículo: Juan Carlos CASTILLO QUIÑONES (2019), "La (de)seguritización del Gobierno Regional del Kurdistán. El movimiento kurdo en Iraq desde 1991" en Revista de Estudios Internacionales Mediterráneos, 27, pp. 46-63.

Para acceder a este artículo: https://doi.org/10.15366/reim2019.27.004

\section{Resumen}

Este artículo examina de forma empírica las interacciones de seguridad entre el Gobierno Regional del Kurdistán y el estado iraquí. La evidencia discursiva muestra cómo a pesar de que la cuestión kurda en Iraq entró en un proceso de gradual normalización política o deseguritización a partir de 1991, al mismo tiempo muchas dimensiones del tema permanecieron seguritizadas. Usamos la figura de las narrativas polarizadoras para mostrar los efectos negativos de estas acciones en el largo plazo. Concluimos que la (de)seguritización ha introducido más violencia en la política iraquí y creado condiciones para la prolongación del conflicto entre los kurdos y el gobierno central en Bagdad.

Palabras clave: kurdos/ seguritización/ deseguritización/ Gobierno Regional del Kurdistán/ Irak/

\section{Abstract}

This paper empirically examines the security interactions between the Kurdistan Regional Government and the Iraqi state. The discursive evidence shows that despite the Kurdish question in Iraq began a steady process of political normalization -desecuritization- since 1991, several dimensions of the issue remained securitized. We use the figure of splitting speech acts to show the long-term negative effects of these actions. We concluded that (de)securitization has

\footnotetext{
1 "Este artículo es posible gracias al apoyo recibido por el Programa de Movilidad que ofrece el Programa de Apoyo a los Estudios de Posgrado (PAEP), en el marco del Doctorado en Ciencias Políticas y Sociales de la Universidad Nacional Autónoma de México."
} 
introduced more violence into Iraqi politics and created conditions for the prolongation of the conflict between the Kurds and the state.

Keywords: Kurds/ securitization/ desecuritization/ Kurdistan Regional Government/ Iraq

\section{Introducción}

El espectro político kurdo del siglo XXI se puede dividir en dos grandes corrientes ideológicas. Por un lado, los grupos que aún mantienen viva la idea de un Estado kurdo, como es el caso del liderazgo kurdo en Iraq, y otros que lo rechazan buscando formas de gobierno no estatal. Este trabajo analiza la historia del movimiento kurdo en Iraq, en relación con el establecimiento del Gobierno Regional del Kurdistán (GRK) como un cuasi Estado o Estado de facto en la región. El colapso del Estado iraquí en 2003 creó estructuras de oportunidad política que permitieron el surgimiento de un nuevo sistema de gobernanza kurdo en el norte de Iraq. Los líderes kurdos ${ }^{2}$ actuaron frente a este contexto de dos formas: buscando reconocimiento internacional y avalando una estrategia que tendió a deseguritizar sus relaciones con el gobierno central en Bagdad y con Turquía, como parte de una estrategia para avanzar en su proyecto de construcción estatal a partir de 1991.

La profunda debilidad del gobierno central iraquí, generada a partir de la crisis del Golfo de 1990, fue el factor crucial que permitió el establecimiento del GRK como una entidad semi independiente en Iraq. El proyecto de construcción estatal que en ese contexto impulsó la administración kurda contó con la protección y asistencia de Estados Unidos (Gunter y Yavuz, 2004: 108). Entre 1991 y 2003, el Kurdistán iraquí, o Kurdistán del sur, se constituyó en lo que muchos académicos definieron como un proto Estado o Estado de facto (Ver Bengio, 2012; Natali, 2010; Stansfield, 2003; Voller, 2014) emanado de la gradual disolución de la autoridad iraquí. El movimiento kurdo en Iraq entró desde entonces en una fase que denominamos aquí de deseguritización. Este concepto deriva de la Escuela de Copenhague (EC), la cual provee, en este caso de estudio, la estructura teórica para discutir las relaciones y asuntos de seguridad entre el GRK y el Estado iraquí.

De acuerdo con los estudios de seguridad de la EC, en cualquier sociedad, sus elites dominantes pueden someter la identidad de un grupo a versiones extremas de politización que denominamos seguritización (Buzan et al, 1998: 24; ver también Kuzemko, 2012). La teoría de la seguritización se basa en identificar qué, cómo y cuándo los gobiernos construyen y nombran un asunto como un peligro existencial para un objeto referencial que puede ser tangible, como la integridad territorial, o intangible, como la nación o la identidad nacional (Buzan et al., 1998). La seguritización, por lo tanto, refiere a situaciones donde el uso de medidas excepcionales es legitimado en contra de un grupo previamente designado como amenaza. Los primeros teóricos concibieron este proceso como indeseable y propusieron como contramedida la estrategia de la deseguritización (Waever, 2000: 253) es decir, el retorno de los asuntos al ámbito de la política normal y de la deliberación pública. Corrientes posteriores vieron en esta propuesta incluso un

\footnotetext{
${ }^{2}$ Cuando hablamos de liderazgos kurdos en Iraq nos referimos a los partidos políticos que dominan la escena en el Kurdistán iraquí; es decir, al Partido Democrático del Kurdistán (PDK), la facción dominante, y a los partidos emanados de su tradición política; en específico, a la Unión Patriótica del Kurdistán (UPK), una escisión del PDK establecida en 1976. En 2009 surgió una tercera fuerza de importancia, que por razones metodológicas no discutiremos en este trabajo, conocida como el movimiento Gorran (Cambio), fundado a partir de una ruptura dentro de la UPK. Estos partidos son los actores principales dentro del territorio autónomo y se encuentran inmersos en una lucha por el poder y control político.
}

REIM № 27 (diciembre 2019) ISSN: $1887-4460$ 
potencial emancipador (Cfr. Aradau, 2004). Sin embargo, este trabajo cuestiona dicha tendencia en definir la deseguritización como algo positivo y deseable. De hecho, como hipótesis de trabajo, esta investigación sostiene que la deseguritización, en la práctica, produce más violencia y crea condiciones para la prolongación y exacerbación de los conflictos.

Utilizando una interpretación revisionista del modelo de seguritización de la EC, este trabajo examina de forma empírica los asuntos de seguridad en Iraq alrededor del tema kurdo desde 1991, aunque hace hincapié en el periodo posterior a 2003. La evidencia discursiva muestra que muchas demandas de los kurdos en Iraq fueron constitucionalmente normalizadas y resueltas a partir de 2003 -deseguritisadas-, al mismo tiempo que otras dimensiones de seguridad que preocupaban a las autoridades kurdas permanecieron politizadas por los diferentes gobiernos iraquíes desde 2006. Debido a esta simultaneidad de prácticas discursivas seguritizadoras y deseguritizadoras, las relaciones entre el GRK y el Estado iraquí han estado plagadas de suspicacia, tensión y violencia. Lejos de constituir una práctica emancipadora, la desegurización transformó las relaciones kurdo-árabes en Iraq en un nuevo dilema de seguridad.

El trabajo se divide en tres apartados. El primero explica las líneas generales del enfoque teórico aplicado en esta investigación y ofrece una discusión que expone algunos de los sesgos que envuelven al enfoque de la deseguritización. El segundo analiza el proceso de politización y seguritización de la identidad kurda en Iraq y en el denominado Medio Oriente a lo largo del siglo XX. El tercer acápite explica el proceso de deseguritización por el que atraviesa el movimiento kurdo en Iraq desde 1991, enfatizando los efectos negativos que esto ha traído consigo a partir de 2003.

\section{La Escuela de Copenhague y la Teoría de Seguritización desde una perspectiva crítica}

La Teoría de la Seguritización (TS) es un programa de investigación que analiza procesos de (de)seguritización, los cuales están compuestos por acciones discursivas que pueden ser de naturaleza seguritizadora o deseguritizadora. La teoría convencional indica que estos movimientos ocurren en temporalidades distintas y exclusivas, con la seguritización ocurriendo antes que la deseguritización de un asunto previamente considerado como amenaza por algún gobierno. Sin embargo, la frecuente simultaneidad con la que ocurren estos dos procesos ha pasado desapercibida debido a un conjunto de sesgos metodológicos, temporales y ontológicos desarrollados dentro de la TS, lo cual no analizaremos aquí pues excede el ámbito de este estudio. Sn embargo, cabe recalcar que la frecuente simultaneidad de los movimientos (de)seguritizadores urge repensar el estatus normativo que recibe la deseguritización dentro de las teorías de seguridad. Aunque la deseguritización es vista tradicionalmente como una normatividad positiva, otros argumentan que, en la práctica, no existe una división temporal en las acciones discursivas, por lo que la inherente promulgación de la deseguritización junto a los movimientos seguritizadores (Austin y Beaulieu-Brossard, 2018) puede introducir más violencia en las políticas de seguridad $y$, de hecho, exacerbar conflictos prolongados.

El modelo de seguritización de la Escuela de Copenhague ofrece el recuento tradicional de este proceso. La seguritización identifica qué, cuándo y cómo un asunto es seguritizado, es decir, su desplazamiento de la esfera de la política normal hacia su constitución como un tema de seguridad. De acuerdo con Buzan et al., en toda sociedad una situación o identidad grupal puede estar no politizada (no es tema de debate público o de formulación de alguna política en específico), politizada (el tema es parte de la política pública y requiere de una decisión gubernamental y de asignación de recursos) o seguritizada, es decir, presentada como una amenaza existencial y sujeta de medidas fuera de la política convencional (1998: 23) ${ }^{3}$. Es decir,

\footnotetext{
${ }^{3}$ La identidad es construida por pueblos y grupos a través de muchos procesos y prácticas políticas. Toda vez que una identidad surge y se sedimenta en una colectividad entra en el campo de la competencia política (Buzan y Waever, 1997: 243), convirtiéndose en un proceso de negociación entre diferentes grupos de interés (McSweeney, 1999: 73).
} 
antes de ser seguritizados, los asuntos públicos son politizados en una primera instancia. El acto de politización permite que una identidad no hegemónica pueda ser tratada "más allá de los límites normales" (Kuzemko, 2012: 130) del procedimiento político y, de acuerdo con Tarimo, las elites politizan a ciertos grupos o minorías en función de intereses políticos y económicos (2010: 304). Bajo ciertas circunstancias la politización de una identidad conlleva a la seguritización de la misma y a su designación como amenaza.

De acuerdo con Buzan, una cuestión de seguridad emerge no por la existencia real de una amenaza o peligro inminente, sino porque el tema es presentado como tal a una audiencia pública mediante actos discursivos (Buzan et al., 1998: 24-25). El proceso de seguritización se basa en identificar los supuestos y creencias colectivas que construyen dicha amenaza. La teoría señala que el traspaso de un asunto de la política normal hacia la esfera de la seguritización requiere de una autoridad (el gobierno iraquí, por ejemplo) que lo nombre como una amenaza existencial para un objeto de referencia (la integridad territorial) o colectivo (la nación iraquí). Buzan y Waever definen la seguritización como un acto discursivo exitoso -llevado a cabo por las autoridades de un gobierno-, por el cual los miembros de una comunidad perciben bajo amenaza algo a lo que colectivamente le atribuyen valor -objeto de referencia- y exigen una respuesta efectiva, por drástica que ésta sea (2003: 358). El movimiento seguritizador se logra sólo cuando las elites de un gobierno consiguen el respaldo de un segmento significativo de la población que avala el uso de medidas extraordinarias en contra de la así nombrada amenaza.

La seguritización es, en este sentido, una gramática de la seguridad que encontramos en artefactos textuales conocidos como actos discursivos (Austin y Beaulieu-Brossard, 2017: 9) elaborados por los actores seguritizadores. Las acciones discursivas etiquetan un asunto como una amenaza existencial y son el canal mediante el cual se legitima el uso de medidas de excepcionalidad en su contra. Floyd menciona que toda seguritización requiere de tres pasos para su implementación exitosa: identificación de la amenaza (movimiento seguritizador), medidas de emergencia (ruptura del estado de derecho) y aceptación del proceso por una audiencia (2007: 42). Para este caso de estudio, un acto seguritizador se lleva a cabo cuando, al argumentar un sentido de prioridad y urgencia, algún agente seguritizador (las elites políticas) nombran a un grupo étnico como un peligro inminente (los kurdos) para un objeto de referencia (la identidad étnica o integridad de la nación) por medio de discursos y narrativas polarizadoras ${ }^{4}$. Desde esta perspectiva, tanto la politización como la seguritización de un asunto son desarrollos intersubjetivos. La identidad kurda fue politizada tras el surgimiento de los Estados-nación en el denominado Medio Oriente y seguritizada a lo largo del siglo XX.

La EC también propuso la noción de la deseguritización como estrategia de retorno de los asuntos seguritizados al ámbito de la normalidad política. La deseguritización fue definida originalmente como un movimiento seguritizador fallido, en el cual la audiencia pública ya no acepta la representación de una situación como amenaza existencial (Waever, 1995: 56), deteniendo o disminuyendo con ello las capacidades del gobierno para justificar medidas de emergencia o estados de excepción. Otros estudios reforzaron la visión de la deseguritización como contrapeso

Con bastante frecuencia, las elites buscan imponer la cultura de la etnia dominante para generar discursos que legitimen su control político. En muchos Estados del Medio Oriente, la politización y el uso de mecanismos de represión en contra de las minorías es utilizado para centralizar el poder mediante el reforzamiento de las instituciones responsables de la reproducción cultural.

${ }^{4}$ McSweeney argumenta que la identidad no es una causa de conflictos [erróneamente tildados como] étnicos, sino un instrumento al servicio de las elites políticas (1996: 86). En otras palabras, la identidad étnica muchas veces es manipulada por elites políticas para obtener ventajas y concesiones políticas y económicas.

REIM № 27 (diciembre 2019) ISSN: $1887-4460$ 
ético y ontológico a las políticas de seguridad excepcional, dando paso a una definición normativa del concepto. Como resultado, estudios recientes sobre los conflictos han planteado la deseguritización como una estrategia óptima para el regreso a la normalidad política y la negociación pacífica. La noción de Aradau (2004) sobre la deseguritización como política emancipatoria fue fundamental en la articulación de esta visión normativa y positiva del concepto.

Bajo el mantra de la emancipación, Claudia Aradau (2004) propone el establecimiento de normas universales aplicables a los grupos excluidos del acceso a derechos, junto con un proceso de desidentificación de la identidad asignada. La deseguritización desde esta perspectiva se convierte en una estrategia para conformar a la sociedad como un espacio despolitizado y trascendental (Behnke, 2006: 63-68). Sin embargo, una estrategia basada en el principio del reconocimiento y la universalidad resulta problemática en la medida que su aplicación sugiere la eliminación de las identidades particulares. En otras palabras, el precio de la emancipación conlleva la eliminación de la diferencia y la inclusión física del individuo su exclusión ontológica, supeditada a un principio universalizante, (Hama, 2015:144).

Posiciones revisionistas de la EC cuestionan estas visiones esencialistas sobre los movimientos desguritizadores, así como su sesgo emancipatorio. Behnke, por ejemplo, argumenta que la deseguritización como acto discursivo es contradictoria. Declarar que un asunto no constituye más una amenaza a la seguridad da paso a un "juego del lenguaje" (2006: 65) del que sacan ventaja los actores más poderosos. Roe sostiene que la vinculación de la deseguritización con la deconstrucción de identidades colectivas entra en directa contradicción con la búsqueda de derechos para las minorías (2005:43), pues refiere principalmente a la preservación de las identidades distintivas.

Los argumentos vertidos arriba permiten al menos cuestionar la tendencia prevaleciente en los estudios de seguridad por definir la deseguritización como algo deseable. Austin y BeaulieuBrossard (2018) desarrollaron la crítica más completa al respecto. Ambos autores estructuran su investigación a partir de tres bases: metodológica, temporal y ontológica. Para este trabajo nos enfocamos en los dos últimos elementos. Los sesgos temporales refieren en cómo el entendimiento de los movimientos seguritizadores como mutuamente exclusivos -uno perteneciendo al ámbito de la excepcionalidad, mientras que el otro a la normalidad política- ha llevado a la ilusión de concebir al último como un derivado del primero. La (de)seguritización, sin embargo, no posee una temporalidad esencialmente lineal. De hecho, los movimientos seguritizadores y deseguritizadores a menudo ocurren al mismo tiempo a través de acciones discursivas polarizadoras. Esta lectura provee de una aproximación más dinámica para examinar las interacciones de seguridad. Siguiendo a Austin, argumentamos que las relaciones entre el movimiento kurdo en Iraq y el gobierno central evidencian un proceso de (de)seguritización. Partimos de la idea de que la deseguritización del movimiento kurdo ocurrió a expensas de Washington tras la formación del gobierno Regional del Kurdistán en 1992, en un momento de máxima seguritización de la cuestión kurda en Iraq. Por lo tanto, la simultaneidad de acciones seguritizadoras y deseguritizadoras, ocurriendo sin un lapso de tiempo específico (Austin y Beaulieu-Brossard, 2018: 315), han sido la norma en las relaciones de seguridad entre el GRK y el gobierno central iraquí.

Los sesgos ontológicos refieren a la esencialización de los diferentes elementos de un proceso (de)seguritizador (es decir, actor seguritizador, objeto de referencia, sujeto y audiencia). Esto es particularmente problemático para el sujeto de referencia -lo que es percibido como amenazaporque seguido se le representa y asume como un ente homogéneo. Estos supuestos se observan a menudo en los movimientos deseguritizadores. Las condiciones para la deseguritización de un grupo, previamente etiquetado como amenaza, se enuncian sin considerar las complejidades y las identidades superpuestas que lo constituyen. Austin y Beaulieu-Brossard utilizan la figura de los actos discursivos polarizadores para ilustrar cómo los movimientos deseguritizadores en la 
práctica se convierten en esfuerzos externos para dividir supuestas amenazas en "partes más o menos amenazantes, que requieren más o menos de medidas extraordinarias" (2018: 315). Bajo este tipo de medidas, los grupos sociales son continuamente redefinidos $y$ artificialmente segmentados entre sectores moderados y radicales, en función del interés de actores externos más poderosos. Al crear esta división, la deseguritización establece las precondiciones para considerar a un segmento de la población como potencialmente reconciliable, mientras que otros sectores "de línea dura" permanecen seguritizados y sujetos de medidas extraordinarias. Este tipo de prácticas discursivas se convierten en una forma clara de violencia.

Lo expuesto en este apartado indica que los movimientos seguritizadores y deseguritizadores reciben un tratamiento separado que conduce a un problema normativo. Esta separación aumenta la expectativa de percibir los resultados de la deseguritización como intrínsecamente positivos, pasando por alto que se promulgan con bastante frecuencia como una imposición externa y artificial hacia los sujetos o grupos etiquetados como amenaza. La deseguritización funciona en los hechos como un instrumento de las elites para segmentar a la sociedad entre "quienes son objeto de seguritización (los de línea dura) y aquellos potencialmente deseguritizables" (Austin y Beaulieu-Brossard, 2018: 302), si cumplen con ciertas condiciones que establecen los actores más poderosos.

De esta forma, los movimientos deseguritizadores son realmente demandas impuestas sobre un sector social considerado como "moderado", a cambio de cierto reconocimiento político. Esta perspectiva evoca bastante bien lo ocurrido con el movimiento kurdo en Iraq a partir de 1991, como consecuencia del apoyo y reconocimiento recibido desde entonces por Estados Unidos y parte de la comunidad internacional. Sin embargo, como veremos más adelante, esta división externa y artificial de una comunidad produce más violencia al crear condiciones para la prolongación de los conflictos.

\section{La seguritización de los kurdos en el Medio Oriente y en Iraq}

La seguritización de la identidad kurda está ligada con los procesos de formación estatal en el Medio Oriente. Al final de la Primera Guerra Mundial, el Tratado de Lausana de 1923 y el llamado Pacto de Mosul de 1926, el cual incorporó de manera formal esa provincia al mandato británico de Iraq, sellaron de forma definitiva el destino de los kurdos. El Kurdistán fue dividido como consecuencia del desmembramiento del Imperio otomano y la identidad étnica de los kurdos suprimida debido a los procesos de construcción nacional en Irán, Iraq, Siria y Turquía. El Kurdistán se convirtió a partir de entonces en lo que el sociólogo Ismail Beşikçi (1990) definió como una colonia internacional, colonizada por diferentes estados del denominado Medio Oriente. Los kurdos fueron conceptualizados como minorías en sus respectivos territorios, subordinados a la etnicidad nacional dominante y seguritizados mediante "una serie de mecanismos de exclusión y negación" (Bozarslan, 2014: 4) ontológica a lo largo del siglo XX. En consecuencia, la historia moderna del Kurdistán está marcada por levantamientos encabezados por diversos movimientos de resistencia en contra de los procesos de formación estatal (ver Jongerden, 2019) y la subsecuente subyugación de los kurdos en las entidades estatales.

La idea del Estado moldeó el proyecto político de muchos de los movimientos kurdos no solo en Iraq, sino en distintos contextos y latitudes del norte de Asia occidental. El surgimiento de un etnonacionalismo kurdo fue la respuesta a una doctrina colonial que definió como civilizadas a aquellas sociedades poseedoras de un Estado-nación. Por lo tanto, no es extraño que en la primera mitad del siglo XX surgieran diversos partidos políticos kurdos que articularon sus programas alrededor del objetivo de una formación estatal, que les garantizase su reconocimiento 
como sociedad moderna. De esta forma, los kurdos buscaron legitimar sus demandas sobre la base del derecho a la autodeterminación. Al igual que con otros pueblos del Medio Oriente, dichos reclamos se expresaron en la búsqueda de formas de autonomía, autogobierno o en el establecimiento de un Estado propio.

Bajo la retórica del tribalismo, los Estados intentaron deslegitimar los reclamos kurdos al postular la identidad kurda como tribal y pre-moderna en esencia. El Estado, en cambio, fue reconocido como el agente garante de la modernización de aquellas entidades calificadas de tribales (Mohammadpour y Soleimani, 2019: 9), por medio de su asimilación o represión. Surgió entonces una dialéctica reproducida en buena parte de la academia que definió a los kurdos en términos de "atraso" frente al avasallante avance del progreso social, encarnado en el Estado y la supresión de las formas de organización local. Tal discurso permitió legitimar prácticas coloniales que etiquetaron como pre-modernos, o tribales, auténticos procesos de resistencia étnica contra la estrecha centralización de los sistemas políticos en construcción ${ }^{5}$. Dichas acciones generaron el ambiente para que los Estados justificasen después un proceso más amplio de seguritización y definición colectiva de los kurdos como amenaza regional.

La formación de una consciencia nacionalista entre los kurdos, y su concreción en partidos políticos, llevó pronto a la politización de su identidad en los incipientes Estados. Espacios púbicos como las escuelas, ciudades con importancia histórica, mitos y narraciones de la cultura popular oral desempeñaron un papel fundamental en la formación política de un sentido de kurdicidad (Gourley, 2017). El Kurdistán se convirtió en un espacio en disputa y en una importante marca simbólica de identidad para los kurdos. Durante las primeras décadas del siglo XX, diversas insurrecciones kurdas trataron de revertir el orden político de la posguerra. Como en el caso de la revuelta de Sheik Mahmoud en 1919 en Iraq, o de Simko en Irán (1918-22), estas primeras rebeliones tuvieron un importante componente anticolonial, anticentralizador y etnonacional. De hecho, el periodo posterior a la Primera Guerra Mundial fue de intensa actividad política en el Kurdistán y en otras partes del Medio Oriente. Las insurrecciones kurdas estimularon el surgimiento de un sentimiento nacionalista, dando paso décadas después a la creación de partidos políticos, y en el caso de Irán, de la efímera República de Mahabad (Soleimani, 2017: 955). Sin embargo, el activismo político kurdo de la primera mitad del siglo XX fue reprimido con éxito hacia finales de 1940 en Irán, y en 1938 en Turquía con el aplastamiento de la revuelta de Dersim.

La naturaleza transnacional de su identidad transformó a los kurdos en un asunto de seguridad transfronteriza (Bozarslan, 2014: 6-8). Turquía, Siria, Irán e Iraq trataron de contener la lucha kurda mediante mecanismos de contención regional como el Pacto de Saadabad de 1937. Este acuerdo inauguró la aproximación estratégica hacia el tema kurdo y derivó en la invención geopolítica del Kurdistán como un espacio atrasado (pre-moderno), fuente de sedición e inseguridades para los Estados. Acuerdos posteriores incluirían a Siria. Más tarde, la Organización de Defensa de Medio Oriente (1951) y el Pacto de Bagdad (1955) fueron diseñados como mecanismos avalados desde Washington para contener cualquier infiltración comunista en la región, aunque en la práctica ambos se utilizaron también para neutralizar a cualquier movimiento guerrillero kurdo transfronterizo.

La permanencia de este enfoque de seguridad hacia los kurdos fue fomentado por los intereses de Occidente durante el periodo de la Guerra Fría. Bajo la lógica de contención de la era bipolar, concepciones de seguridad con un enfoque militar (ver Bilgin, 2005), dominaron la región y priorizaron el mantenimiento de la estabilidad de los regímenes aliados en el Medio Oriente. Sin embargo, más que disuadir el expansionismo soviético, los Estados receptores del apoyo

\footnotetext{
${ }^{5}$ Mohammadpour y Soleimani señalan que argumentar en contra de la aplicación de categorías ideológicas como tribu, tribal o tribalismo no significa negar la contingencia histórica de las relaciones comunales o de los sistemas de parentesco en la región del Medio Oriente, sino cuestionar la supuesta aplicabilidad universal de esos términos (Mohammadpour y Soleimani, 2019).
} 
estadounidense, bajo el mantra de la lucha contra el comunismo, dirigieron sus recursos para reprimir o eliminar cualquier elemento interno percibido como amenaza, incluido el etnonacionalismo kurdo.

En el caso particular de Iraq, la historia moderna de los kurdos está plagada de tensión y conflictos con las autoridades centrales. La ausencia de una justa representación en las instituciones de toma de decisiones del estado, así como las exigencias de autogobierno en las áreas pobladas en su mayoría por kurdos derivó en una serie de revueltas desde los primeros años de fundación del Estado iraquí (ver Hasan, 2018). Políticas centralizadoras durante el periodo monárquico (1921-58) y republicano (58-2003) debilitaron la confianza de los kurdos en los sucesivos gobiernos e instituciones estatales. La centralización fue explotada por los gobiernos en Iraq para eliminar a la oposición y negar autonomía a las poblaciones kurdas, lo cual llevó eventualmente al establecimiento de una dictadura y un sistema autoritario en el periodo de Saddam Hussein.

Desde la ocupación de Mosul por las fuerzas británicas en 1918, los kurdos buscaron preservar una serie de compromisos autonómicos establecidos bajo supervisión de la oficialidad británica. Estos acuerdos fueron demolidos primero por los británicos y luego por la monarquía iraquí. La negación del poder mandatario a las exigencias kurdas de autogestión y derechos políticos derivó en sucesivas rebeliones kurdas entre 1920 y 1932, lideradas por Sheikh Mahmud Barzinji (Amery, 1929: 221). Como reacción al uso de la fuerza para neutralizar sus demandas, los kurdos recurrieron a la formación de movimientos políticos y en 1946 surgió el Partido Democrático del Kurdistán (PDK), a instancias de la aristocracia kurda de los Barzani. Desde inicios de 1960 diversas rebeliones declararon la guerra al Estado iraquí lideradas por el PDK y Mahmud Barzani. Aunque las relaciones entre los kurdos y el gobierno central durante la etapa republicana (1958-2003) se alternaron en periodos de insurrección y tregua, la política general de todos los gobiernos iraquíes a las demandas de autonomía étnica fue su definición como una amenaza para la integridad del Estado, definido por los nacionalistas en Bagdad como una nación exclusivamente árabe. A partir de 1963, El Estado iraquí inició una campaña sistemática de arabización de las zonas kurdas (Anderson y Stanfield, 2004: 156) que empujó a más kurdos a la resistencia armada. En este periodo los kurdos también establecieron lazos informales con el Estado de Israel, como una forma de contrarrestar las condiciones geopolíticas adversas y alcanzar un entendimiento con Estados Unidos ${ }^{6}$.

Décadas de seguritización y lucha política de los kurdos resultaron en el reconocimiento de una autonomía territorial kurda en 1970 por el gobierno central de Bagdad. Los acuerdos de Autonomía Territorial de marzo de 1970 estipulaban que una región kurda debía establecerse en las áreas donde los kurdos constituyan una mayoría y sus demarcaciones precisas se determinarían mediante un censo. Las áreas censadas se incorporarían posteriormente a la región autónoma. Las negociaciones también abarcaron temas de representación proporcional de los kurdos en el gobierno central, reforma agraria y, lo más importante para los kurdos, la distribución de los recursos energéticos del gobernorado de Kirkuk. Sin embargo, la desconfianza mutua entre

\footnotetext{
${ }^{6}$ Los contactos entre el Kurdistán iraquí e Israel comenzaron en 1964 por medio de la figura de Kamaran Ali Badirkhan. Israel canalizó ayuda militar en gran escala a los kurdos entre 1960 y 1970 durante las sucesivas guerras en contra del gobierno central iraquí en esa década (Entesar, 2009: 161), forjando una alianza que perduraría en lo sucesivo y sería explotada con fines geopolíticos por Israel y los Barzani. Dicho apoyo fue reconocido públicamente por Israel hasta 1980 durante el gobierno de Menachem Begin. Entre 1965 y 1970, unidades de inteligencia israelí, en cooperación con el Shah de Irán fueron clave en el establecimiento y entrenamiento de un cuerpo de inteligencia kurdo denominado Parastin y responsable de la entrega de equipo militar soviético confiscado a Egipto y Siria en las sucesivas guerras de Israel con los Estados árabes (ver Khosravi et al, 2016).
}

REIM № 27 (diciembre 2019) ISSN: $1887-4460$ 
las partes pronto reemplazó a las expectativas iniciales. Finalmente, el acuerdo fracasó en 1974 debido a la negativa del gobierno de incorporar el gobernorado de Kirkuk a la jurisdicción de la región autónoma.

Barzani reanudó las hostilidades y solicitó ayuda de Estados Unidos, Israel e Irán. En la medida que avanzó la insurgencia, los kurdos recibieron apoyo de la $\mathrm{ClA}$, quienes encontraron en Barzani un aliado dentro de Iraq para equilibrar el respaldo que Bagdad recibía de los soviéticos. Irán también se involucró en el conflicto y desde 1975 incrementó el abastecimiento de armas para las milicias kurdas peshmerga. La insurgencia kurda sobrevivió hasta la firma de los Acuerdos de Argel entre el Shah y Saddam, en calidad de vicepresidente de Iraq. El gobierno iraní se comprometió a reforzar sus fronteras y cesar toda ayuda a los peshmerga, a cambio de garantías por parte de Iraq sobre la fijación de límites en la disputada cuenca fluvial de Shatt al-Arab. Washington también retiró el apoyo a Barzani y la revuelta kurda colapsó de inmediato. Lo anterior resultó en la derrota efectiva del movimiento kurdo y en su escisión en 1975.

Las sucesivas campañas de arabización (Ibíd.,: 53), junto con los horrores que se suscitaron en la década de los ochenta durante la campaña Anfal ${ }^{7}$, marcaron un punto de quiebre en el proceso de politización de la sociedad kurda. Los kurdos se sintieron con más razón una nación oprimida y separada del resto de Iraq, volcándose hacia sus líderes en la aspiración de obtener un Estado propio. Los líderes kurdos utilizaron la kurdicidad como el principal motor de movilización de las masas alrededor de su programa político. Sin embargo, el evento paradigmático del siglo XX para el liderazgo kurdo sobrevino en el contexto de la crisis del Golfo de 1990-91. El establecimiento de una autonomía kurda de facto en el norte de Iraq inauguró un proceso de deseguritización del tema kurdo, el cual contó con el aval de Estados Unidos y parte de la comunidad internacional.

\section{La deseguritización de la cuestión kurda en Iraq: contexto y cambios en el escenario regional posterior a 1991}

Como se mencionó antes, entre 1921 y 1991 el Estado iraquí seguritizó y reprimió de forma sistemática a los kurdos. Sin embargo, esta condición cambió con el establecimiento del Gobierno Regional del Kurdistán (GRK). La gradual deseguritización de ciertas dimensiones del tema kurdo en Iraq comenzó con el establecimiento de una zona de exclusión aérea en las áreas kurdas al norte del paralelo 36. La derrota de Iraq en Kuwait en 1991 provocó otra rebelión masiva de los kurdos que pronto fue aplastada por las fuerzas de seguridad. La crisis de refugiados generada en el Kurdistán justificó la intervención de Estados Unidos para crear un enclave aliado en el norte de Iraq, con la implementación de un refugio seguro para los kurdos. El retiro de las unidades del ejército iraquí de las áreas kurdas causó un vació administrativo que permitió a los kurdos establecer en 1992 el GRK. Esta nueva entidad territorial, nacida bajo el cobijo de Washington, abarcó los gobernorados - provincias- de Sulamaniyah, Erbil y Dohuk ${ }^{8}$ (Jogerden, 2019: 63) y quedó bajo la jurisdicción conjunta del PDK y la Unión Patriótica del Kurdistán ${ }^{9}$.

En el contexto posterior a 2003, la deseguritización del GRK significó un proceso de normalización del tema kurdo en Iraq en el plano constitucional, y a nivel regional de acomodamiento de los Estados frente a la existencia de una entidad autónoma kurda. Sin embargo, el reconocimiento oficial del GRK en la Constitución de 2005 transformó las relaciones entre las autoridades kurdas y el gobierno central en un nuevo dilema de seguridad. La deseguritización de los partidos kurdos en Iraq evidenció efectos negativos en el largo plazo, pues muchas demandas de los kurdos continuaron politizadas en extremo por los diferentes gobiernos iraquíes desde 2006.

\footnotetext{
${ }^{7}$ Entre estos horrores se cuentan los ataques químicos y las deportaciones masivas de 1987-88 en Halabja y otras poblaciones kurdas, en el contexto de la guerra entre Irán e Iraq de 1980-88 (Jongerden, 2019: 64; HRW, 1993).

${ }^{8}$ Partes de la provincia de Nínive encima de la zona de exclusión aérea fueron incorporadas a la provincia de Dohuk y el norte de la provincia de Diyala fue absorbida por Sulaymaniyah.

${ }^{9}$ La Unión Patriótica del Kurdistán surgió como una escisión del PDK fundada por Jalal Talabani en el contexto de la crisis derivada de la derrota de la rebelión kurda en 1975. Ver infra.
} 
La relación del GRK con el gobierno israelí también contribuyó a tensar las relaciones entre Erbil y Bagdad, aumentando las acusaciones hacia los kurdos de buscar establecer un segundo Israel en el Medio Oriente. La presencia informal de unidades israelíes en el Kurdistán incrementó en el contexto posterior a 2003 como resultado del debilitamiento del gobierno central iraquí. Conducido en buena medida por su aislamiento político en la región, el gobierno israelí ha intento crear divergencias políticas y de seguridad para minar a sus adversarios regionales. Las expresiones públicas israelíes de apoyo a la independencia del Kurdistán iraquí buscan en buena medida influir también en los kurdos de Irán, y convertir al GRK en un enclave desde el cual promover el federalismo y con ello desestabilizar al régimen de Teherán.

La deseguritización del tema kurdo en Iraq ocurrió a partir de un doble proceso que denominamos de estabilización y reemplazo. La estabilización de un asunto refiere a la disminución en su percepción como amenaza debido a ciertos factores contingentes, como cambios dramáticos en el contexto regional o internacional. El reemplazo, en cambio, significa el desplazamiento de un tema de la esfera política de la excepcionalidad, al tiempo que otro tema o dimensiones del mismo permanecen seguritizados (verHansen, 2012). El establecimiento del GRK desde 1992 provocó la estabilización de ciertas dimensiones del tema kurdo en Iraq. Dichas dimensiones fueron el reconocimiento institucional de sus liderazgos y de ciertos territorios del Kurdistán iraquí, así como el establecimiento de canales de interlocución con los sectores considerados moderados. Sin embargo, otros planos de la cuestión kurda permanecieron manejadas como asuntos de seguridad. En particular, el tema de Kirkuk y los territorios en disputa fueron replanteados como una amenaza potencial para la integridad territorial iraquí.

El movimiento deseguritizador más visible fue quizá la estabilización del liderazgo kurdo en Iraq, el cual pasó de un movimiento guerrillero "amenazante" a constituir un proyecto institucionalizado "deseguritisable". Los primeros intentos de institucionalización fueron bloqueados por el estallido de una guerra civil entre el PDK y la UPK de 1994 a 1998, pero retomados después tras la firma de un acuerdo de paz mediado por Estados Unidos. Los dos partidos políticos establecieron sendos acuerdos de reparto del poder y se embarcaron en un proceso de construcción estatal en las áreas autónomas bajo su control. Al dar prioridad a la negociación por encima del conflicto, ambos partidos adaptaron sus estrategias a las demandas de los actores externos, principalmente de Washington y de Ankara, asegurando con ello el acceso a ingresos y reconocimiento a través de la ayuda internacional.

La invasión estadounidense de Iraq alentó el proceso de construcción estatal kurdo. A partir de 2003, Estados Unidos y los medios internacionales definieron a los kurdos como aliados regionales en la lucha contra el extremismo islámico y socios clave para contener la influencia iraní en Iraq. El sistema político posterior al Ba'ath transformó radicalmente las interacciones kurdas con los actores regionales, tras el reconocimiento del GRK en la Constitución iraquí de 2005. Para evitar colisionar con los intereses estadounidenses, los formuladores de políticas en Iraq y Turquía se vieron obligados a incorporar algunas de las exigencias del GRK en sus agendas de seguridad y aceptar la existencia formal de una entidad autónoma kurda.

\section{Narrativas polarizadoras y sus efectos en el proceso de (de)seguritización del movimiento kurdo en Iraq}

Como se mencionó antes, las narrativas polarizadoras son estrategias discursivas que buscan dividir artificialmente la realidad social entre segmentos moderados y radicales o asuntos negociables y líneas rojas (Austin and Beaulieu-Brossard, 2018). La apertura de condiciones para la deseguritización del tema kurdo generó una retórica de segmentación regional que dividió 
discursivamente a los kurdos entre amigas o enemigos. Mientras que el GRK y sus liderazgos fueron reconocidos como aliados de Occidente, otros movimientos kurdos de alcance transregional, como el Partido de los Trabajadores del Kurdistán (PKK) en Turquía, permanecieron bajo la etiqueta de terroristas. En el caso de Iraq, la desguritización generó zonas de tensión y ambigüedad alrededor del tema de Kirkuk, el petróleo y la implementación del artículo 140 de la Constitución.

La deseguritización del GRK ocurrió mediante su construcción discursiva como un bastión de estabilidad en medio de un vecindario hostil y antidemocrático, lo cual le valió su reconocimiento internacional en el contexto posterior a 1991. Las estrategias discursivas articuladas para la estabilización del GRK fueron declaradas por miembros de la comunidad internacional y por las propias autoridades kurdas. Estos movimientos deseguritizadores impulsados por el propio GRK utilizaron las narrativas del 'experimento democrático' (Barzani 2008; Kurdistan Presidency, 2009), la 'victimización' (KRG Council of Ministers, 2015; Baser y Toivanen, 2017; KRG UK Representation, 2018a, b) y 'el liberalismo' (UK Parliament, 2014) para cabildear en los países occidentales a favor de su reconocimiento como gobierno legítimo. En un comentario para el Wall Street Journal, el entonces presidente del GRK, Masud Barzani, declaró lo siguiente:

"Estoy orgulloso de que la Región del Kurdistán sea a la vez un modelo y una puerta de entrada para el resto de Irak. Nuestro difícil camino hacia una democracia federal secular está inspirado por los EE. UU. (...) Estamos orgullosos de lo que la región del Kurdistán es hoy: una sociedad civil próspera en el corazón de Medio Oriente" (Barzani, 2008).

La impronta de las campañas de represión en 1988 -Anfal y el genocidio en Halabja- otorgó a los kurdos y sus elites suficiente legitimidad para justificar sus reclamos de autonomía y garantías de preservación de sus derechos en Iraq. La expansión de su sector petrolero, así como la capacidad para proveer seguridad en las áreas kurdas permitió a las autoridades en Erbil generar un discurso de gobernanza democrática y de atracción de inversiones en los campos petroleros del Kurdistán. Esta imagen del GRK fue magnificada por el boom económico que vivió la región a finales de los noventa y hasta 2014 (Jüde, 2017). Dicha proyección fue el motor principal del proceso deseguritizador que presentó al GRK como un aliado de Occidente. Al tiempo que importantes dimensiones del tema kurdo eran deseguritizadas en Iraq, las medidas militares de Turquía en contra del PKK no cesaron y, desde mediados del 2000, las incursiones militares en el norte de Iraq se volvieron una constante, avaladas incluso por las autoridades del GRK.

Los primeros pasos para deseguritizar las políticas del GRK fueron llevados a cabo por Estados Unidos y Turquía a mediados de 1990. La estabilidad en la región kurda se convirtió en un componente de los objetivos de política exterior de Washington hacia el Medio Oriente y parte de la política energética y de seguridad de Ankara. Las autoridades turcas presentaron al GRK desde la segunda mitad de la década del 2000 como un socio comercial, desplazando la tradicional retórica de seguridad y de amenaza hacia el autonomismo kurdo en Iraq. La transformación del GRK en un aliado clave de Estados Unidos desde 2003 fue fundamental en este cambio de narrativa. La llegada al poder del Partido Justicia y Desarrollo (AKP) en 2002 en Turquía, y su reelección en 2007, también fue decisivo en ese sentido, al traducirse en una reducción importante del papel del establishment militar kemalista en el diseño de la política exterior turca.

El acelerado crecimiento económico turco de la década del 2000 y los intentos de adhesión a la Unión Europea durante los primeros años de administración del AKP generaron el contexto propicio para el cambio de narrativa hacia la cuestión kurda (Altunışık, 2011). Las necesidades energéticas de Turquía convirtieron al GRK en una fuente más barata de hidrocarburos y en una alternativa atractiva para romper la dependencia del sector empresarial turco frente a los energéticos rusos e iraníes (Tol, 2014). Sin embargo, el establecimiento de relaciones de Turquía con el GRK estuvo siempre condicionado por el compromiso de las autoridades kurdas con la integridad territorial de Iraq y de una autonomía sin Kirkuk. Analistas cercanos a Ankara 
elaboraron una retórica que presentaba estos intercambios como benéficos para las tres partes involucradas:

"Se espera que los ductos [en el norte de Irak] generen ingresos, aumenten la interdependencia y sirvan para afianzar la paz y la estabilidad entre Turquía e Iraq, y también sirvan para la unidad dentro de Iraq, ya que tanto los kurdos como los chiitas [de Iraq] se beneficiarían del acuerdo" (Yetkin, 2013).

Al cooperar con Erbil, los estrategas en Turquía buscaron direccionar la conducción política del GRK en beneficio de sus intereses estratégicos. Ankara intentó hacer del GRK una entidad satélite, totalmente dependiente del gobierno turco en términos económicos, de acceso al mercado global y en un aliado en la lucha contra el PKK. Las autoridades turcas utilizaron la deseguritización del liderazgo kurdo de Iraq como una estrategia de reemplazo en sus esquemas de seguridad frente a su propio "problema" kurdo. El gobierno turco emprendió una fuerte campaña discursiva polarizadora al respecto, difundiendo narrativas que mostraban a un PKK radical y violento frente a un sector kurdo moderado y socio comercial representado por el GRK. Estas narrativas permitieron al gobierno turco justificar su postura hacia el tema kurdo, argumentando una posición no anti-kurda sino anti-PKK.

El GRK buscó adaptarse a las demandas turcas, haciendo eco de los discursos y temas que demandó la agenda de seguridad de Turquía. En declaraciones oficiales y entrevistas, los miembros del GRK señalaron su desaprobación hacia las operaciones militares del PKK en territorio bajo jurisdicción del GRK. Barzani declaró en su momento: "Usaremos todos los métodos, incluida la presión para disuadir al PKK de lanzar ataques transfronterizos [desde territorio del GRK]" (Ekurd 2012). El Ministerio de Asuntos Peshmerga destacó que "...el PKK debe cesar sus acciones desestabilizadoras [porque] aumentan las tensiones en el área [y debe] permitir que la vida regrese a la normalidad en la región" (KRG Cabinet, 2016); en tanto, el entonces primer ministro kurdo, Nechirvan Barzani, señaló como condición sine qua non para la paz que "[el PKK] necesita entender que este problema no puede solucionarse por medio de la guerra y la violencia" (KRG Cabinet 2017). Sin embargo, la implementación de las condiciones deseguritizadoras impuestas por Ánkara sólo terminó por erosionar las bases de legitimidad de las autoridades del GRK. La escisión discursiva del PKK tuvo un costo político elevado para Masud Barzani, frente a un amplio sector que observó como la causa kurda fue sacrificada por el presidente del GRK, al avalar la tesis colonialista del "kurdo bueno y el kurdo malo".

El incremento en la actuación del GRK como una entidad semi independiente entre 2005 y 2014 generó temores entre las elites políticas de Bagdad. El tema kurdo en Iraq fue deseguritizado a nivel constitucional tras la caída del régimen baathistaen y la instauración de un sistema federal, pero las autoridades iraquíes rápidamente buscaron revertir el esquema de autonomía obtenido por los kurdos en ese contexto. La Constitución iraquí de 2005 incluyó en el Artículo 140 el reconocimiento oficial de la Región del Kurdistán de Iraq como una entidad autónoma. Además, dicho artículo proporcionó una fórmula política y legal para resolver las demandas históricas de los kurdos con respecto a territorios reclamados por los nacionalistas kurdos que fueron arabizados en la década de 1980, incluyendo la provincia petrolera de Kirkuk. La ruta constitucional fijada para ello estableció un proceso de normalización (el retorno de las personas que habían sido expulsadas o desplazadas de las provincias kurdas antes de 2003), un censo y referendos locales para decidir la pertenencia de las áreas en disputa al gobierno central o al Kurdistán. Otra demanda histórica fue la inclusión de la lengua kurda como idioma oficial en el Artículo 4. La 
Constitución también permitió al GRK mantener sus propias milicias armadas peshmerga, reconociéndolas como las fuerzas de seguridad en la región del Kurdistán (Hasan 2015: 148-155).

Aunque la mayoría de los derechos y demandas kurdas fueron reconocidos de manera formal en la Constitución iraquí, las relaciones entre las elites políticas iraquíes y kurdas estuvieron plagadas de temor y suspicacia (Hama, 2015: 148). De hecho, en la práctica el tema kurdo no se trasladó totalmente a la esfera de la normalidad política. Lo que ocurrió en Iraq fue un proceso (de)seguritizador, en el cual ciertas dimensiones de la cuestión kurda fueron objeto de negociación, mientras que otras causaron tensión y conflictos entre Erbil y Bagdad. La cuestión de Kirkuk y la implementación del artículo 140 evidenció la naturaleza simultánea de estos movimientos (de)seguritizadores, al resolver en el plano constitucional el tema kurdo, al mismo tiempo que otros asuntos relacionados con las zonas estratégicas en disputa fueron politizados en extremo.

El estancamiento y la parálisis política caracterizaron las relaciones kurdo-árabes entre 2006 y 2014. El gobierno iraquí usó el tema de Kirkuk como una narrativa polarizadora para convertir el debate político sobre la centralización y el federalismo en Iraq en un asunto de secesión, justificando con ello su falta de compromiso para encontrar una solución política a los temas que causaban fricción con las autoridades kurdas. Las crecientes tendencias autoritarias del gobierno central encabezado por al Maliki (2006-2014) convirtieron gradualmente la disputa entre el GRK y el gobierno central en un juego de suma cero, donde ninguna de las partes estuvo dispuesta a comprometerse en una ronda seria de negociaciones. Diversas fuerzas políticas dentro de Iraq comenzaron a cuestionar la validez del artículo 140, argumentando que garantizaba la consolidación de un proyecto cuasi estatal para el GRK. Al respecto, un líder del bloque político dominante (con una agenda confesional pro chiíta) concluyó lo siguiente: “...el federalismo sobre una base sectaria desatará otros reclamos y...demandas de [autonomía] de otras minorías religiosas... para sus propios distritos federales. Todo esto servirá sólo para debilitar y dividir a Irak, y por eso lo rechazamos" (ICG, 2009: 17).

Pronto apareció en Iraq un sector político revisionista que demandó reescribir la Constitución para acotar las capacidades autónomas de los kurdos. Estos grupos políticos encabezados por el primer ministro al Maliki declararon que la Constitución se estableció en un contexto de profunda fragilidad del Estado iraquí y bajo la influencia de Estados Unidos (Behner, 2005). Dicha corriente argumentó que el amplio esquema federal diseñado para Iraq constituía una amenaza existencial para la unidad nacional. El problema fue que esas razones no fueron argüidas por los sectores democráticos dentro de Iraq, sino por las fuerzas dominantes del nuevo régimen que buscaban recentralizar el poder político. Al-Maliki puso de manifiesto esa postura al señalar que, "la Constitución fue redactada de forma apresurada y en condiciones extraordinarias. [Ésta] limita los poderes del gobierno central y tememos que el federalismo perjudique al país" (KRG cabinet, 2008)"

Por su parte, los kurdos continuaron demandando la implementación de las disposiciones incorporadas en la Constitución, incluida una estructura política descentralizada para Iraq. No obstante, las autoridades kurdas nunca generaron un discurso de rechazo a la unidad iraquí, sino que veían en el federalismo la garantía para preservar la autonomía política, económica y cultural del Kurdistán. Al respecto, las autoridades del GRK declararon en su momento: "La Constitución... especifica la distribución de poderes (regionales y federales). La región del Kurdistán no busca más poder del que le otorga la Constitución. Solo se busca que la Constitución se aplique" (KRG Cabinet, 2017). Iraq entró así en una guerra de narrativas comunales y el discurso político terminó etnitizando las relaciones kurdo-árabes Los derechos otorgados por la constitución a los kurdos fueron presentados de nuevo como una amenaza existencial para la soberanía iraquí. Hanan Saeed al-Fatlawi, destacada activista política y en su momento diputada cercana al oficialista 
Partido Dawa, proporcionó pruebas para respaldar esta afirmación en el siguiente extracto de una entrevista para Dijlah TV en 2014:

"La historia escribirá que las elites políticas chiitas protegieron la unidad del Estado iraquí con sus dientes, de la misma forma que hemos protegido la unidad del Estado iraquí durante diez años; trabajamos muy duro para evitar que las áreas en disputa (Kirkuk y Mosul) regresen al Kurdistán" (citado en Hama, 2015: 150).

En la práctica, el proceso (de)seguritizador exacerbó las percepciones de seguridad entre las autoridades kurdas e iraquíes. La implementación del artículo 140 se entendió como una amenaza existencial que atentaba contra la unidad de Iraq. En tanto, el GRK vio el fracaso en su implementación como un peligro para su autonomía política y para la permanencia del esquema federal en Iraq. El gobierno iraquí asumió que resolver el problema mediante lo estipulado en la constitución (normalización y referéndum) daría lugar a la pérdida de Kirkuk y pondría en riesgo la viabilidad económica del Estado iraquí. La mayor parte del petróleo en Iraq se produce y extrae de Kirkuk (Barkey y Laipson, 2005) por lo que su traspaso a la jurisdicción kurda fue equiparado con la fragmentación de todo el país. A juicio de este autor, el verdadero objetivo del gobierno iraquí fue utilizar el tema de Kirkuk como un justificante para recentralizar el poder político y desechar la formula federal en construcción desde 2005. Para los kurdos, en cambio, Kirkuk fue definido como una parte histórica e indivisible de la nación kurda. El líder kurdo y luego presidente iraquí, Jalal Talabani, caracterizó Kirkuk como "la Jerusalén del Kurdistán" (Ekurd, 2011) y Barzani (2003) afirmó que "si los kurdos reclaman... Kirkuk, no es porque sea una ciudad rica en petróleo... sino porque estas ciudades...son una parte importante de la historia kurda".

La confrontación discursiva entre Erbil y Bagdad derivó en la práctica en la militarización efectiva de los territorios en disputa desde 2008 y alcanzó marcados tintes etnosectarios en 2014 por las acciones de ambos bandos. En 2014, el GRK ocupó por la fuerza la mayoría de las áreas en disputa para evitar su caída en manos de las milicias del Estado islámico o Daesh. EL GRK enseguida emprendió un proceso de kurdificación de los nuevos territorios bajo su control, con las fuerzas peshmerga expulsando a residentes árabes de confesión sunnita o impidiendo su regreso a distritos y villas cercanas a Mosul, como es el caso de Hamdaniya (HRW, 2019). Las tensiones se exacerbaron cuando Kirkuk estuvo bajo control de las milicias kurdas peshmerga y al Maliki encuadró dicha acción bajo la narrativa de una declaración de guerra, acusando al GRK de formar parte de una conspiración externa para permitir a Daesh avanzar sobre Bagdad (MEMO, 2014).

Estas pugnas tomaron otro matiz luego de la celebración del referéndum en pro de la independencia kurda de 2017. La convocatoria a un referéndum constituyó una línea roja no sólo para Iraq, sino para Turquía e Irán. En una acción concertada con Ánkara y Teherán, el gobierno iraquí desplegó a diversas milicias pro iraníes para expulsar a los kurdos de Kirkuk y de la mayoría de los territorios en disputa (Kamaran, 2019). El entonces primer ministro iraquí Haider al Abadi describió la operación como "...una operación iraquí dirigida a restaurar la autoridad federal en áreas que estaban fuera del control gubernamental desde 2014" (al-Abadi, 2017). Estos desarrollos dejaron de manifiesto que, a pesar de la deseguritización constitucional del tema kurdo en Iraq, los diferentes gobiernos iraquíes politizaron la implementación del artículo 140, creando con ello impedimentos para su ejecución. En los hechos, el despliegue de fuerzas militares iraquíes y kurdas en las áreas en disputa terminó por convertir la agenda de intercambios políticos entre el GRK y el gobierno central en un nuevo dilema de seguridad.

\section{Conclusión}

REIM № 27 (diciembre 2019) ISSN: $1887-4460$ 
Después de la Primera Guerra Mundial y hasta 1991, el Estado iraquí reprimió y seguritizó a los kurdos de forma sistemática. La seguritización de los kurdos en Iraq y en el Medio Oriente se construyó a partir de discursos y prácticas del Estado que equipararon el reconocimiento de la diferencia étnica con la desintegración de la nación. En consecuencia, la etnicidad surgió como un aspecto definitorio de la política iraquí. El recuento histórico muestra que a lo largo del siglo XX el Kurdistán se convirtió en un espacio de intenso activismo, lo cual derivó en la formación de diversos movimientos políticos que buscaron transgredir las fronteras que separaban a los kurdos. A partir de 1991, el movimiento kurdo en Iraq entró en una fase de institucionalización, la cual inició un proceso de deseguritización y un cambio en la narrativa que definía a los kurdos como amenaza.

Los análisis expuestos en las secciones previas de este trabajo permiten cuestionar la validez de asumir la deseguritización del tema kurdo en Iraq como un proceso positivo en esencia. Lejos de llevar a una resolución pacífica de las controversias entre las autoridades kurdas y el gobierno central, el gradual proceso deseguritizador del GRK introdujo más violencia y tensión dentro de la política iraquí, pues significó la imposición externa y artificial de una estrategia política que deseguritizó a ciertos segmentos de la sociedad kurda, pero excluyó a otros sectores y asuntos de este proceso. A pesar de que las demandas de los kurdos en Iraq fueron deseguritizadas en el plano constitucional, la politización y militarización del tema de Kirkuk acabó por transformar el conjunto de relaciones entre el GRK y el gobierno iraquí en un dilema de seguridad.

Esta aproximación abre la posibilidad de entender las aristas que han rodeado a la cuestión kurda en Iraq durante las últimas décadas de una forma más dinámica y compleja. En la práctica vemos que nunca ocurrió una despolitización completa del tema kurdo, sino una deseguritización selectiva de ciertas dimensiones que interesaban a los actores más poderosos, como Turquía o Estados Unidos. La asimetría de este proceso se evidencia en la serie de demandas y expectativas que debió de cumplir el GRK, desde 1991, a cambio de obtener cierto reconocimiento político. Tales exigencias incluyeron la fragmentación artificial de la comunidad y la implementación de mecanismos que exacerbaron la violencia en Iraq.

Más allá de su importancia académica, la perspectiva aquí planteada mostró los potenciales efectos negativos que pueden generar los procesos deseguritizadores en el largo plazo. Lo anterior ofrece al lector un entendimiento más profundo de los mecanismos subyacentes en conflictos prolongados o crisis sociopolíticas recurrentes, como es el caso de la relación histórica entre los kurdos y el Estado en Iraq.

\section{Referencias}

AITUNISIK, B.M. y MARTIN, L.G. (2011): "Making Sense of Turkish Foreign Policy in the Middle East Under AKP", Turkish Studies, no 12, Vol 4, pp. 569-587. DOI: https://doi.org/10.1080/14683849.2011.622513

AL-ABADI, Haider: "Iraq Will Remain United", The New York Times, 18 de octubre de 2017, disponible en

https://www.nytimes.com/2017/10/18/opinion/iraq-will-remain-united.html? $r=0$ [consulta: 6 de noviembre de 2018].

AMERY, L. (1929): "Political survey of Iraq", The National Archive, Reference: CAB 24/201/29, disponible en http://discovery.nationalarchives.gov.uk/details/r/D7727972 [consulta: 25 de septiembre de 2016].

ANDERSON, L.D. y STANFIELD, Gareth (2004): The future of Iraq: dictatorship, democracy, or division?, Nueva York, Palgrave Macmillan.

ARADAU, C. (2004): "Security and the democratic scene: De-securitization and emancipation", Journal of International Relations and Development, no 7, Vol. 4, pp. 388-413. DOI: https://doi.org/10.1057/palgrave.jird.1800030 
AUSTIN, J. Y BEAULIEU-BROSSARD, P. (2018): "(De)securitisation dilemmas: Theorising the simultaneous enaction of securitisation and desecuritisation", Review of International Studies, no 44, (2), pp. 1-23. DOI: https://doi.org/10.1017/S0260210517000511

BARKEY, H. y LAIPSON, E. (2005): "Iraqi Kurds and Iraq's Future. Middle East", Policy, no 12, Vol. 4, pp. 66-76. DOI: https://doi.org/10.1111/j.1475-4967.2005.00225.x

BARZANI, Masud: "Iraqi Kurdish claim for federalism: A Kurdish-Arab Partnership", Khabat, 21 de diciembre de 2003, disponible en http://www.kurdnet.net/www.kdp.info/ [consulta: 20 de abril de 2018].

BARZANI, Masud: "Kurdistan is a Model for Iraq", The Wall Street Journal, 12 de noviembre de 2008 disponible en https://www.ws].com/articles/SB122645258001119425 [consulta: 20 de julio de 2018].

BASER, B. y TOIVANEN, M. (Junio de 2017): "The politics of genocide recognition: Kurdish nationbuilding and commemoration in the post-Saddam era", Journal of Genocide Research, n 19, Vol 3, pp. 1-23. DOI: https://doi.org/10.1080/14623528.2017.1338644

BEHNKE, A. (2006): "No Way out: Desecuritization, Emancipation and the Eternal Return of the Political. A Reply to Aradau", Journal of International Relations and Development, no 1, vol. 9, pp. 62-69. DOI: https://doi.org/10.1057/palgrave.jird.1800070

BEHNEER, L.: "Why Sunnis Don't Support Iraq's Constitution", Council of Foreign Relations, 2005, disponible en: https://www.cfr.org/backgrounder/why-sunnis-dont-support-iraqsconstitution [Consulta 20 de junio de 2019].

BENGIO, O (2012): The Kurds of Iraq: Building a State within a State, Boulder, CO: Lynne Rienner. BESIKCI, I. (1990): International Colony Kurdistan, Londres, Ediciones Parvana.

BILGIN, P. (2005): Regional security in the Middle East. A critical perspective, Nueva York, Routledge. DOI: https://doi.org/10.4324/9780203358320

BOZARSLAN, H. (2014): "The Kurds and Middle Eastern "state of violence": the 1980s and 2010s", Kurdish Studies, no 2, Vol. 1, pp. 4-13. DOI: https://doi.org/10.33182/ks.v2i1.376

BUZAN, B. Y WAEVER, O. (1997): "Slippery? Contradictory? Sociologically Untenable? The Copenhagen School Replies", Review of International Studies, no 2, pp. 241-250. DOI: https://doi.org/10.1017/S0260210597002416

--- (2003): Regions and Powers: The Structure of International Security, Nueva York, Cambridge University Press.

---, B.O., WAEVER y J. Wilde (1998): Security: A New Framework for Analysis, Londres, Pinter.

EKURD Daily: "Talabani criticized for designating Kirkuk 'Jerusalem of Kurdistan', MP says it's 'serious'", Ekurd, 10 de marzo de 2011, disponible en http://ekurd.net/mismas/articles/misc2011/3/kirkuk666.htm [consulta: 21 de junio de 2019].

EKURD Daily: "Masoud Barzani says won't allow PKK to operate from Iraqi Kurdistan", 20 de abril de 2012, Ekurd, disponible en http://ekurd.net/mismas/articles/misc2012/4/turkey3893.htm [consulta: 20 abril de 2018].

FLOYD, R. (2007): "Human Security and the Copenhagen School's Securitization Approach: Conceptualizing Human Security as a Securitizing Move", Human Security Journal, no. 37, Vol 5, pp. 38-49.

GOURLEY, William (2017): "Kurdayetî: Pan-Kurdish Solidarity and Cross-Border Links in Times of War and Trauma", Middle East Critique, DOI: https://doi.org/10.1080/19436149.2017.1411110 GUNTER, M. y H. Yavuz (2004): "The Kurds in Iraq: Why Kurdish Statehood Is Unlikely", Middle East Policy, no 1, vol. 11, pp. 106-131. DOI: https://doi.org/10.1111/j.1061924.2004.00145.x 
HANSEN, L. (2012): "Reconstructing desecuritisation", Review of International Studies, no 38, Vol. 3, pp. 525-46. DOI: https://doi.org/10.1017/S0260210511000581

HAMA, H. (2015): "Politization of Kurdish Security In Iraq since 2003", Jadavpur Journal of International Relations, no. 19, Vol. 2, pp. 137-158. DOI: https://doi.org/10.1177/0973598416639414

HASAN, Jalal (junio de 2018): "The Kurds in Iraq: Problem and Solution Attempts", Journal of Political and Security Studies, no 1, Vol. 1, pp. 127-156. DOI: https://doi.org/10.31271/jopss.10005 HUMAN RIGHTS WATCH: "Genocide in Iraq. The Anfal Campaign Against the Kurds", Middle East Watch Report, julio de 1993, disponible en https://www.hrw.org/reports/1993/iraqanfal/ [consulta: 20 de agosto de 2017].

HUMAN RIGHTS WATCH: "Kurdistan Region of Iraq: Arabs Not Allowed Home", 6 de septiembre de 2019, disponible en: https://www.hrw.org/news/2019/09/06/kurdistan-region-iraq-arabs-notallowed-home [consulta: 23 de noviembre de 2019].

INTERNATIONAL CRISIS GROUP (2009): "Iraq and the Kurds: trouble along the trigger line", Report 88, Middle East and North Africa, disponible en https://www.crisisgroup.org/middle-east-northafrica/gulf-and-arabian-peninsula/iraq/iraq-and-kurds-trouble-along-trigger-line [consulta: 28 de mayo de 2019].

JONGERDEN, Joost (2019): "Governing Kurdistan: Self-Administration in the Kurdistan Regional Government in Iraq and the Democratic Federation of Northern Syria", Ethnopolitics, no 18, Vol 1, pp. 61-75. DOI: https://doi.org/10.1080/17449057.2018.1525166

JÜDE, J. (2017): "Contesting borders? The formation of Iraqi Kurdistan's de facto state" International Affairs, no 93, Vol. 4, pp. 847-63. DOI: https://doi.org/10.1093/ia/iix125

KAMARAN Palani Et al. (2019): "The development of Kurdistan's de facto statehood: Kurdistan's September 2017 referendum for independence", Third World Quarterly, DOI:

https://doi.org/10.1080/01436597.2019.1619452

KHOSRAVI, Jamal Et.al. (2016): "The Presence of Israel in Iraqi Kurdistan and its Security Challenges for Iran's National Security", Journal of Politics and Law, no 7, Vol. 9, pp. 169-177, DOI: https://doi.org/10.5539/jpl.v9n7p169

KRG Council of Ministers: "Statement on the 27th anniversary of Anfal Campaign", KRG Austria, 15 de abril de 2015, disponible en http://austria.krg.org/en/stellungnahme-des-krg-ministerrats-zum27-jahrestag-der-anfal-kampagne/ [consulta: 20 de noviembre de 2018].

KRG UK Representation: "30th Commemoration of Kurdish Genocide was held at UK Parliament", 19 de marzo de 2018a, disponible en http://www.uk.gov.krd/30th-commemoration-of-kurdishgenocide-was-held-at-uk-parliament/ [consulta: 19 de mayo de 2019].

KRG UK Representation: "KRG UK High Representative's speech at the 30th Commemoration of Kurdish Genocide", 21 de marzo de 2018, disponible en http://www.uk.gov.krd/krg-uk-highrepresentatives-speech-to-the-30th-anniversary-commemoration-of-kurdish-genocide/ [consulta: 19 de mayo de 2019].

KURDISTAN PRESIDENCY: "Statement in Response to Amnesty International Report", 20 abril de 2009, disponible

en

http://cabinet.gov.krd/a/d.aspx?a=28956\&r=\&area $=60 \& s=02010000 \& \mathrm{I}=12 \& \mathrm{~s}=010000$ [consulta: 22 de septiembre de 2018].

KUZEMKO, C. (2012): Dynamics of Energy Governance in Europe and Russia, Londres, Palgrave Macmillan. DOI: https://doi.org/10.1057/9780230370944

MCSWEENEY, B. (1996): "Identity and Security: Buzan and the Copenhagen School", Review of International Studies, no 1, Vol. 22, pp. 81-93. DOI: https://doi.org/10.1017/S0260210500118467 MIDDLE EAST MONITOR (MEMO): "Maliki's coalition: Kurdish annexation of Kirkuk is a declaration of war", 30 de junio de 2014 disponible en: https://www.middleeastmonitor.com/20140630malikis-coalition-kurdish-annexation-of-kirkuk-is-a-declaration-of-war/ [consulta: 5 de noviembre de 2018]. 
MOHAMMADPOUR, Ahmad y SOLEIMANI, Kamal (2019): "Interrogating the tribal: the aporia of 'tribalism' in the sociological study of the Middle East", The British Journal of Sociology, no 0, Vol. 0, pp. 1-26, DOI: https://doi.org/10.1111/1468-4446.12656

NATALI, D. (2010): The Kurdish Quasi-State: Development and Dependency in Post-Gulf War Iraq, Syracuse, Syracuse University Press.

ROE, P. (2005): Ethnic Violence and the Societal Security Dilemma, Londres y Nueva York, Routledge, DOI: https://doi.org/10.4324/9780203005446

SOLEIMANI, Kamal. (2017): "The Kurdish image in statist historiography: the case of Simko", Middle Eastern Studies, no 53, Vol. 6, pp. 949-965, DOI: https://doi.org/10.1080/00263206.2017.1341409

STANFIELD, G. (2003): Iraqi Kurdistan: Political Development and Emergent Democracy, Londres, Routledge Curzon.

TARIMO, Aquiline (2010): "Politicization of ethnic identities: The case of contemporary Africa", Journal of Asian and African Studies, no 3, Vol 45, pp. 297-308, DOI: https://doi.org/10.1177/0021909610364775

TOL G.: "Untangling the Turkey-KRG Energy Partnership: Looking Beyond Economic Drivers". Global Turkey in Europe series, Policy Brief, 14 de marzo de 2014, disponible en: http://www.iai.it/sites/default/files/GTE PB 14.pdf [consulta: 15 de mayo de 2019].

UK Parliament: "UK Government Policy on the Kurdistan Region of Iraq", Foreign Affairs, 2014, disponible

en:

https://publications.parliament.uk/pa/cm201415/cmselect/cmfaff/564/56409.htm\#n76 [consulta:

21 de marzo de 2019].

VOLLER, Y. (2014): The Kurdish Liberation Movement In Iraq From insurgency to statehood, Nueva York, Routledge, DOI: https://doi.org/10.4324/9781315886954

WAEVER, Ole (1995): "Securitization and de-securitization", en LIPSCHUTZ, R. (ed.) On Security, Nueva York, Columbia University Press, pp. 46-86.

YETKIN, M.: "Kurdish dreams might come true, through oil pipelines", Hurriyet Daily News, 16 de noviembre de 2013, disponible en http://www.hurriyetdailynews.com/opinion/muratyetkin/kurdish-dreams-might-come-true-through-oil-pipelines-57994 [consulta: 12 de mayo de 2019]. 